\title{
Arthroscopic SLAP Ilb Repair Using Knot-tying Versus Knotless Suture Anchors: Is There a Difference?
}

\author{
Justin W. Arner, MD¹, Joseph De Groot, MD², James P. Bradley, MD²
}

${ }^{1}$ Department of Orthopaedic Surgery, University of Pittsburgh Medical Center, Pittsburgh, PA, USA, ${ }^{2}$ University of Pittsburgh Medical Center, Pittsburgh, PA, USA.

Objectives: SLAP Ilb surgical outcomes have traditionally been less predictable when compared to other shoulder injuries. Traditional knotted anchors may partially be to blame by abrading the rotator cuff leading to tearing and pain.

Methods: Seventy-four athletes who underwent arthroscopic SLAP IIb repair with traditional $(n=42)$ and knotless anchors $(n=32)$ at minimum 2-year follow-up were evaluated. Demographic, surgical data, return to play (RTP), KJOC, ASES, stability, ROM, strength, and pain scores were compared.

Results: Knotless anchors had slightly higher RTP (93.5\%, $58.1 \%$ same level) vs. traditional $(90.2 \%, 53.7 \%$ same level), but was not statistically significant. Knotless anchors were less likely to require revision surgery ( $9 \%)$ compared to traditional anchors (17\%), but was not statistically significant. There was no difference in KJOC, ASES, stability, ROM, strength, and pain between the two anchor types (Table 1). Pain was the only variable linked to decreased RTP $(p<0.0001)$. Younger patients had significantly poorer KJOC $(p=0.02)$ and ASES scores $(p=$ 0.02 ) but no difference in RTP. No difference in outcome measures or RTP was found with gender, age, overhead athletes, number of anchors, or sport type (Table 2). Average follow-up was 6.5 years.

Conclusion: Knotless anchors required less revision surgery, had higher RTP, ASES, and KJOC scores; however, statistical significance was not achieved in this relatively small cohort. Further evaluation, such as case matching and assessment of a larger sample size should be done. Possible other advantages such as tensioning and surgical time may also be important when considering lower profile knotless fixation.

The Orthopaedic Journal of Sports Medicine, 6(7)(suppl 4)

DOI: $10.1177 / 2325967118 S 00107$

CThe Author(s) 2018 